\title{
Sensory protection to enhance functional recovery following proximal nerve injuries: current trends
}

\author{
Boa Tram Nghiem, Ian C. Sando, Yaxi Hu, Melanie G. Urbanchek, Paul S. Cederna \\ Department of Surgery, Section of Plastic and Reconstructive Surgery, University of Michigan Health System, Ann Arbor, \\ MI 48109, USA.
}

Address for correspondence: Dr. Paul S. Cederna, Department of Surgery, Section of Plastic and Reconstructive Surgery , University of Michigan Health System, Ann Arbor, MI 48109, USA. E-mail: cederna@med.umich.edu

\begin{abstract}
Proximal nerve injury can lead to devastating functional impairment. Because axonal regeneration is slow, timely reinnervation of denervated muscle does not occur. These denervated muscles atrophy and lose function. Sensory protection is a surgical technique thought to prevent denervated muscle impairment using local sensory nerves to provide trophic support to the muscle until motor nerves can regenerate, and neuromuscular junctions are reestablished. We performed a comprehensive literature search using multiple databases to find primary articles reporting on the outcomes and treatment of sensory protection. This paper reviews the three main approaches to sensory protection: (1) end-to-end neurorrhaphy, (2) end-to-side neurorrhaphy, and (3) direct muscle neurotization. It discusses the evidence supporting each technique and outlines goals for future investigations.
\end{abstract}

Key words:

Denervation, muscle, nerve, neurorrhaphy, protection, regeneration, sensory

\section{INTRODUCTION}

Approximately, $65 \%$ of peripheral nerve injuries occur in the upper extremity. Healthy males between the ages of 18 and 35 are most commonly affected and the majority of peripheral nerve injuries are caused by trauma or malignant disease. ${ }^{[1]}$ Axonal regeneration is slow, and there is a critical window for muscle reinnervation before the denervated muscle becomes permanently impaired..$^{[2]}$ Two months after injury, the denervated muscle exhibits reduced motor units but does not demonstrate changes in muscle fiber. ${ }^{[3,4]}$ After 6 months, however, the muscle experiences irreversible muscle atrophy and weakness. ${ }^{[4,5]}$ If primary repair cannot reestablish motor endplate connections within this critical

\begin{tabular}{|l|l|}
\hline \multicolumn{2}{|c|}{ Access this article online } \\
\hline Quick Response Code: & Website: \\
\hline & www.parjournal.net \\
\cline { 2 - 3 } & \\
\hline & \\
\cline { 2 - 3 } & \\
\hline
\end{tabular}

window, one should consider alternative approaches to protect the muscle before irreversible structural or functional impairments occur.

When nerve transection is not amenable to primary tensionless neurorrhaphy, the gold standard for repair is early nerve reconstruction using autologous nerve grafting. ${ }^{[6]}$ This method is not always feasible, however, due to delays in operative management, limitations of the donor nerve, including insufficient graft length or diameter, or morbidity to the donor site. Alternative approaches to early autologous nerve repair include use of decellular xenografts, synthetic grafts, or sensory protection.

Sensory protection is used to prevent denervated muscle from atrophy and subsequent functional loss. Temporarily protecting denervated muscle or "babysitting" it with a nearby branch of a motor nerve successfully maintains the muscle viability. ${ }^{[7]}$ At a second surgery, the babysitter nerve is replaced with a nerve with the needed control once that residual end has elongated, and neurorrhaphy can be performed..$^{[2,8]}$ Similar babysitting with a sensory nerve is also a way to maintain muscle viability. ${ }^{[9]}$ A sensory nerve is coapted to the motor nerve stump in close proximity 
to the muscle for maintenance of muscle health while the proximal motor nerve is regenerating. ${ }^{[9-11]}$ A donor sensory nerve is thought to provide trophic support to the denervated muscle until the native motor axon is able to regenerate and reinnervate its target. ${ }^{[12]}$ In essence, sensory protection provides an interim protective effect on the denervated muscle prior to surgical nerve reconstruction.

We performed an extensive literature search using PubMed, Ovid, and Embase databases using keywords "sensory", "nerve", "protection”, "regeneration", and "denervation" to find primary articles reporting on the treatment and outcomes of the sensory protection either in humans or animal models. This paper discusses the three main approaches to sensory protection and reviews the literature for each. We set the framework for future studies and advocate for further investigation of sensory protection in the upper extremities.

\section{MUSCLE DENERVATION AND REINNERVATION}

\section{Nerve injury and muscle denervation}

The peripheral nervous system has a remarkable capacity for regeneration following nerve injury. When a peripheral nerve is severed, it undergoes Wallerian degeneration and triggers a cascade of biochemical changes allowing future regrowth. Muscle fibers maintain viability immediately following denervation, however, atrophic changes such as reabsorption of myofibrils, shrinkage of muscle cells, and expansion of the extracellular matrix with collagen rapidly commence following denervation. ${ }^{[13]}$ Proteases play a role by promoting axonal degeneration, macrophage infiltration, and myelin degradation in damaged nerves. ${ }^{[14]}$

Without prompt reinnervation, myofibril disorganization, and later mosaic disappearance marks imminent muscle fiber cell death. ${ }^{[15]}$ Prolonged denervation leads to muscle fiber necrosis, connective tissue hyperplasia, decreased vascularity, and depletion of satellite cells needed for regeneration. ${ }^{[4,9,16]}$ Further, denervated muscles become less receptive to regenerating motor axons due to the loss of neurotransmitters, neurotrophic factors, and viable muscle cells. ${ }^{[2,17]}$ These structural changes significantly impact the muscle's contractile properties. The decrease in cross-sectional area of muscle fibers translates to a reduction in maximum tension generated by tetanic muscle contractions. Later on, myofibril disorganization and collagenization diminishes specific force capacity (force per physiological cross sectional unit of muscle). From a functional perspective, maximum tension, specific force, and power all progressively decrease with time. ${ }^{[13]}$

\section{Nerve regeneration and muscle recovery}

Regenerative processes occur synchronously with degradative mechanisms to ensure maximal recovery. Axonal regeneration occurs at a rate of $1 \mathrm{~mm} / \mathrm{day}$ and is affected by age, nerve type, and grade of injury. ${ }^{[18]}$ Recovery involves axonal growth, synapse formation, and restoration of contractile properties. Schwann cells play an essential role in regrowth by increasing regenerative proteins, including growth factors and adhesion molecules, to create a growth-rich milieu. ${ }^{[19]}$ In addition, native endoneurial conduits guide the reestablishment of neuromuscular connections. Research shows that preservation of the original motor endplates is essential for precise contact, synaptic differentiation, and maintenance of reestablished neural connections. ${ }^{[20,21]}$ This growth-supportive environment is significantly diminished if reinnervation does not occur in a timely manner. ${ }^{[2]}$ Although the exact timeframe is debatable, Sulaiman and Gordon $^{[22]}$ proposed a 4-week window for nerve repair, after which the motor neuron has diminished ability to regenerate axons into the distal nerve stump.

Both time and distance limit spontaneous reinnervation of muscles. When immediate nerve reconstruction is not possible, sensory protection is the most effective means of providing temporary trophic support to prevent muscle degeneration. The three surgical techniques for sensory protection include: (1) end-to-end neurorrhaphy, (2) end-to-side neurorrhaphy, and (3) direct muscle neurotization. Nerve transfers with end-to-end neurorrhaphy or end-to-side neurorrhaphy are the most commonly used approaches for sensory protection. End-to-end neurorrhaphy joins the ends of a transected motor nerve and sensory nerve while end-to-side neurorrhaphy connects the end of a transected donor sensory nerve to the side of the injured motor nerve stump. Neurotization, the third and least favored approach, is the direct implantation of a divided sensory nerve into the belly of a denervated muscle. Figure 1 illustrates each technique.

\section{SENSORY PROTECTION - THREE APPROACHES}

\section{End-to-end neurorrhaphy}

End-to-end neurorrhaphy is the classic approach for sensory protection. Bain et al..$^{[9]}$ demonstrated the positive effects of sensory protection on the architecture and

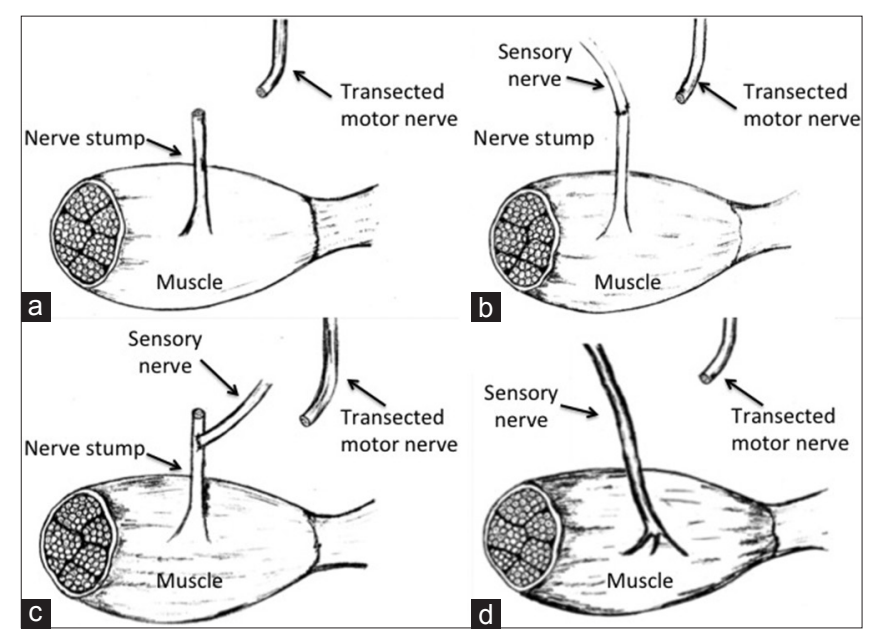

Figure 1: Schematic representation of surgical methods for sensory protection. (a) Transected nerve, denervated muscle without sensory protection; (b) sensory protection by end-to-end neurorrhaphy; (c) sensory protection by end-to-side neurorrhaphy; (d) sensory protection by direct muscle neurotization 
function of the gastrocnemius and soleus muscles following denervation. The sensory-protected group underwent saphenous-to-tibial nerve transfer using end-to-end repair. Compared to the unprotected group, gross and histological examination showed the sensory-protected group had higher muscle weight and greater preservation of muscle structure, including less fiber atrophy and connective tissue hyperplasia. More importantly, the sensory-protected rats demonstrated larger maximum compound action potentials and relative preservation of isometric force overtime. ${ }^{\mid 9]}$ Follow-up studies showed sensory protection also prevented muscle spindle deterioration. ${ }^{[23]}$ Nonetheless, rats that underwent immediate nerve repair had the best structural and functional outcomes.

The benefits of sensory protection have been substantiated by other groups. ${ }^{[24-26]}$ Common outcomes of end-to-end sensory nerve grafting in the lower limbs include preservation of fiber distribution, maintenance of motor endplates, and less muscle atrophy, fibrosis, collagenization, and fat deposition. ${ }^{[16,25]}$ Consistent with lower extremity studies, Beck-Broichsitter et al..$^{[1]}$ found that sensory-protection in the upper extremity resulted in higher muscle weight, larger axon diameter, and larger nerve fiber surface area. However, there was no definitive difference in grasping strength between the sensory-protected and unprotected groups. ${ }^{[1]}$

The protective effect by a purely sensory nerve can be explained by a number of factors. First, the trophic effect of the sensory nerve provides a supportive milieu for the maintenance of skeletal muscles. More specifically, sensory protection modulates the expression of both glial cell line-derived neurotrophic factor (GDNF) and brain-derived neurotrophic factor (BDNF), thereby optimizing the environment for muscle preservation and reinnervation. ${ }^{[12,27]}$ Second, the sensory nerve helps maintain the architecture of the residual nerve stump and basal lamina of endoneurial tubes, thus facilitating later access by regenerating motor axons. ${ }^{[4,28]}$ Third, Schwann cells of the distal stump switch from a myelinating phenotype to a growth-supporting phenotype by upregulating specific genes associated with regeneration. ${ }^{[19]}$ These trophic effects help maintain a growth-supportive environment, which would otherwise deteriorate with time.

Although the research overwhelmingly supports the benefits of sensory protection, it is important to recognize points of contention. Sulaiman et al. ${ }^{[2]}$ proposed that the presence of sensory nerves instead create an unfavorable environment that reduces motor axonal regeneration and myelination of regenerated axons. The authors advocated that sensory nerves actively inhibit motor axonal regeneration by not only physically occupying the endoneurial pathways, but by altering Schwann cells in the distal nerve stump. Specifically, sensory nerves shift Schwann cells toward a phenotype that is less receptive to regenerating motor axons, in part by down-regulating the expression of L2/HNK-1 required for interaction between Schwann cells and motor axons. ${ }^{[29,30]}$ Furthermore, the investigators argue that the functional outcome of chronic denervation is primarily determined by its effect on the injured nerve stump and secondarily by its effect on the muscle. ${ }^{[29]}$ Other studies have not largely substantiated these claims.

\section{End-to-side neurorrhaphy}

End-to-side neurorrhaphy provides trophic support from the donor nerve and enables the regenerated motor axons to reach their target, thus bypassing the need for a second operation to replace the motor nerve supply. In contrast, end-to-end neurorrhaphy requires cutting the donor sensory nerve and suturing it to its native stump once the motor nerve has regenerated, essentially denervating the muscle twice. Studies have shown that two cycles of denervation and reinnervation lead to suboptimal functional recovery, as demonstrated by reduced muscle power and force. ${ }^{[31]}$

End-to-side coaptation is a compelling alternative when conventional end-to-end coaptation is not practical. Such cases occur when the transected end of the motor nerve stump is far from its muscle target or when there are multiple denervated muscles. Zuijdendorp et al. ${ }^{[32]}$ recently investigated the efficacy of sensory protection using end-to-side neurorrhaphy. The investigators sutured the divided end of the sural nerve to the lateral aspect of the tibial nerve stump and examined the gastrocnemius muscle at 5 weeks and 10 weeks postoperatively. Compared to primary end-to-end neurorrhaphy, the sensory-protected group demonstrated a statistically significant increase in muscle weight and decrease in muscle atrophy compared to the unprotected group. ${ }^{[32]}$ Despite the ongoing debate over the efficacy, end-to-side neurorrhaphy remains a viable approach that requires further investigation.

Researchers have also used the end-to-side neurorrhaphy model to study the protective effects of mixed nerve containing both motor and sensory axons. The use of mixed nerve is supported by a recent study by Li et al. . $^{[3]}$ who compared muscle protection following denervation using the peroneal nerve (mixed protection) or sural nerve (sensory protection). They showed that both the mixed- and sensory-protected groups demonstrated preservation of muscle architecture and better functional recovery following reinnervation compared to the unprotected group. Further, the investigators showed that mixed protection was superior to sensory protection in terms of axon structure (more regenerated myelinated axons, larger axonal diameter, thicker myelin sheath) and function (greater contraction force). ${ }^{[3]}$ They controlled for stump reinnervation by the motor component of the mixed nerve by performing end-to-side coaptation and capping the end of the transected motor nerve. In contrast, another study by Michalski et al. ${ }^{[27]}$ reported no difference between mixed- and sensory-protected groups in terms of number of regenerating axons, axon diameter, and myelin cross-sectional area in the distal stump. However, there was a difference in the expression of denervation-induced GDNF, and BDNF expression between the two groups, which suggests that the main benefit of mixed protection is more rapid normalization of trophic factors. 
More researches are needed to elucidate the exact mechanism of sensory protection and compare its functional outcomes with mixed protection. Goals for future studies include comparing differences in Schwann cell phenotype and neurotrophic expression between sensory protection and mixed protection. While mixed protection appears more advantageous compared to sensory protection, the need to sacrifice innervation to a donor muscle to harvest mixed nerve may preclude its use clinically.

\section{Side-to-side neurorrhaphy}

As an extension of end-to-side neurorrhaphy, researchers have also examined the efficacy of performing side-to-side neurorrhaphy. The technique involves joining the side of an intact donor nerve with the side of an injured nerve using a nerve "bridge" composed of either synthetic conduit or autologous graft. The few basic science and clinical studies investigating this technique have yielded mixed results. Experimentally, Shea et al..$^{[34]}$ demonstrated the benefits of side-to-side neurorrhaphy using a synthetic collagen bridge to connect a healthy peroneal nerve with a transected tibial nerve in a rodent model. The investigators noted superior muscle preservation (higher muscle weight and less histologic evidence of muscle damage) and improved functional outcome (gait assessment) in their side-to-side nerve bridge group compared to denervated controls. However, no rats in the experimental group showed axonal regeneration along the length of the entire conduit. Clinically, Magdi Sherif and Amr ${ }^{[35]}$ showed the potential effectiveness of using autologous nerve graft bridges between median and ulnar nerve fascicles at the wrist in patients with high median or ulnar nerve injuries. However, this was a small case series without rigorous outcome measures making it difficult to definitively state that this technique is clinically efficacious.

Although the process of axonal repopulation and end-organ reinnervation is similar between end-to-side and side-to-side neurorrhaphy, the efficacy of side-to-side neurorrhaphy is less. Without donor axon, disruption and injury to the recipient nerve, the neurotrophic signals stimulating Schwann cell proliferation and axonal sprouting through nerve bridges are substantially diminished. ${ }^{[33,36,37]}$ As a result, few axons are available downstream for reinnervation or to provide trophic factors. Thus, compared to other techniques, side-to-side neurorrhaphy is a relatively inefficient technique for nerve reconstruction, and it does not result in clinically significant functional recovery or offer sufficient "protection" from muscle atrophy.

\section{Direct muscle neurotization}

Neurotization showed limited success in early human studies. ${ }^{[38,39]}$ Poor outcomes are in part due to the failure to form new neuromuscular junctions. ${ }^{[40]}$ Specifically, directly implanting a sensory nerve into muscle forces axonal regeneration to occur outside of native endoneurial conduits. The endoneurial conduit is important in regeneration because Schwann cells and basal lamina of the endoneurial sheath produce key components that promote growth (e.g. collagen, fibronectins, and laminin) and provide a substrate for reinnervation. ${ }^{[16,41-43]}$ Impaired reinnervation translates into reduced capacity to generate force. ${ }^{[44]}$

Recent neurotization experiments using animal models have been more promising. Wang et al. ${ }^{[24]}$ demonstrated that implantation of either a sensory nerve or preganglionically avulsed sensory nerve could slow muscle atrophy. Compared to unprotected controls, the implantation groups demonstrated higher fibrillation potential, muscle weight, cross sectional area, and protein content at one and three months after neurotization. In parallel experiments, Ochi et al. ${ }^{[45,46]}$ showed that joining the isografted dorsal root ganglia to the common peroneal nerve stump also mitigated muscle atrophy. Microscopy demonstrated sensory neuron survival and growth of fine axonal branches into the muscle. Although the sensory axons did not reinnervate motor endplates, the neurotized group showed functional advantages over unprotected controls with higher twitch tension and tetanic contraction. Similar to other techniques, the protective effect of neurotization is attributed to trophic factors derived from or stimulated by the sensory nerve. Compared to neurorrhaphy, however, the structural and functional results of direct muscle neurotization remain inferior.

Clinically, neurotization has limited applicability for patients with multiple denervated muscles, including those with proximal median or ulnar nerve injuries. For example, with proximal median nerve transection, over ten muscles in the arm may be affected. Each denervated muscle would require implantation with a separate sensory nerve, rendering this approach impractical. Thus, direct muscle neurotization is only appropriate for a selective group of patients.

\section{FUTURE DIRECTIONS}

To date, the outcomes of peripheral nerve manipulations such as end-to-end and end-to-side neurorrhaphy have primarily been assessed using histomorphometric analysis. Inferences about functional recovery have been made using anatomic measurements such as axonal density and myelin thickness. However, anatomic proxies for nerve regeneration offer little information about functional recovery. ${ }^{[47]}$ For years, muscle contractile properties, such as twitch force, tetanic force, peak-to-peak tension, and contractile velocity have been used to evaluate the degree to which motor axons reestablish their functional connections with muscle. Further studies measuring contractile properties as they relate to sensory protection would provide a more direct measure of muscle integrity and nerve regeneration. In addition, when compared with supra-physiological measures like tetanic force, functional outcomes such as grip strength, ambulation biomechanics, and upper extremity performance tests provide clinically relevant information about the extent and quality of muscle reinnervation. 
Bain et $a l^{[10]}$ set the stage for future clinical trials when they reported the first significant clinical application of sensory protection in a patient with complete sciatic nerve palsy and profound distal denervation following a total hip arthroplasty. The patient underwent end-to-side grafting of fascicles of the saphenous nerve to the motor nerve to the gastrocnemius and the deep branch of the peroneal nerve, thereby protecting the gastrocnemius and tibialis anterior muscles, respectively. One year after surgery, the patient demonstrated notable functional recovery of the gastrocnemius and anterior tibialis muscles based on electrophysiological testing and showed improved performance with activities of daily living. In comparison, none of the unprotected muscles below the knee showed electrophysiological or clinical improvement. Given the degree of sciatic nerve injury, the timing and extent of his improvements could not have been attributed to spontaneous recovery alone. Thus, this sentinel case offers a convincing argument for further clinical trials.

\section{CONCLUSION}

Based on its consistently favorable results in animal models, we advocate for the clinical application of sensory protection in upper limb injuries. Although end-to-end neurorrhaphy has produced the most favorable results, end-to-side coaptation may circumvent the need for a second neurorrhaphy procedure and it avoids multiple episodes of denervation. This effect becomes especially important in proximal injuries with diffuse muscle denervation. Neurotization can be a salvage technique in select patients when the proximal nerve stump is completely avulsed, and nerve transfer is not feasible. Together, these techniques offer hope for patients with peripheral nerve injuries in whom primary repair cannot reestablish motor endplate connections in sufficient time, thus bypassing the need for a second operation.

\section{REFERENCES}

I. Kreiger N, Kelsey JL, Harris C, Pastides H. Injuries to the upper extremity: patterns of occurrence. Clin Plast Surg 198I;8:13-9.

2. Aydin MA, Mackinnon SE, Gu XM, Kobayashi J, Kuzon WM Jr. Force deficits in skeletal muscle after delayed reinnervation. Plast Reconstr Surg 2004; I 13:1712-8.

3. Finkelstein DI, Dooley PC, Luff AR. Recovery of muscle after different periods of denervation and treatments. Muscle Nerve 1993;16:769-77.

4. Fu SY, Gordon T. Contributing factors to poor functional recovery after delayed nerve repair: prolonged denervation. J Neurosci 1995; 15 (5 Pt 2):3886-95.

5. Gutmann E, Young JZ. The re-innervation of muscle after various periods of atrophy. J Anat 1944;78(Pt I-2): 15-43.

6. Evans GR. Peripheral nerve injury: a review and approach to tissue engineered constructs. Anat Rec 200I;263:396-404.

7. Mersa B, Tiangco DA, Terzis JK. Efficacy of the "baby-sitter" procedure after prolonged denervation. J Reconstr Microsurg 2000;16:27-35.

8. Terzis JK, Tzafetta K. The "babysitter" procedure: minihypoglossal to facial nerve transfer and cross-facial nerve grafting. Plast Reconstr Surg 2009; | 23:865-76.

9. Bain JR, Veltri KL, Chamberlain D, Fahnestock M. Improved functional recovery of denervated skeletal muscle after temporary sensory nerve innervation. Neuroscience 200I; 103:503-10.

10. Bain JR, Hason Y, Veltri K, Fahnestock M, Quartly C. Clinical application of sensory protection of denervated muscle. J Neurosurg 2008; 109:955-61.
II. Beck-Broichsitter BE, Becker ST, Lamia A, Fregnan F, Geuna S, Sinis N. Sensoric protection after median nerve injury: babysitter-procedure prevents muscular atrophy and improves neuronal recovery. Biomed Res Int 20I4;20।4:724I97.

12. Zhao C, Veltri K, Li S, Bain JR, Fahnestock M. NGF, BDNF, NT-3, and GDNF mRNA expression in rat skeletal muscle following denervation and sensory protection. J Neurotrauma 2004;21:I468-78.

13. Lien SC, Cederna PS, Kuzon WMJr. Optimizing skeletal muscle reinnervation with nerve transfer. Hand Clin 2008;24:445-54, vii.

14. Liu H, Kim Y, Chattopadhyay S, Shubayev I, Dolkas J, Shubayev VI. Matrix metalloproteinase inhibition enhances the rate of nerve regeneration in vivo by promoting dedifferentiation and mitosis of supporting schwann cells. J Neuropathol Exp Neurol 2010;69:386-95.

15. Borisov $A B$, Carlson BM. Cell death in denervated skeletal muscle is distinct from classical apoptosis. Anat Rec 2000 I;258:305-I8.

16. Veltri K, Kwiecien JM, Minet W, Fahnestock M, Bain JR. Contribution of the distal nerve sheath to nerve and muscle preservation following denervation and sensory protection. J Reconstr Microsurg 2005;21:57-70.

17. Irintchev A, Draguhn A, Wernig A. Reinnervation and recovery of mouse soleus muscle after long-term denervation. Neuroscience 1990;39:231-43.

18. Fex S, Thesleff S. The time required for innervation of denervated muscles by nerve implants. Life Sci 1967;6:635-9.

19. Mirsky R, Jessen KR. The neurobiology of Schwann cells. Brain Pathol 1999;9:293-3। I.

20. Bader D. Reinnervation of motor endplate-containing and motor endplate-less muscle grafts. Dev Biol 1980;77:3 I5-27.

21. Sanes JR, Marshall LM, McMahan UJ. Reinnervation of muscle fiber basal lamina after removal of myofibers. Differentiation of regenerating axons at original synaptic sites. J Cell Biol 1978;78: 176-98.

22. Sulaiman OA, Gordon T. Effects of short- and long-term Schwann cell denervation on peripheral nerve regeneration, myelination, and size. Glia 2000;32:234-46.

23. Elsohemy A, Butler R, Bain JR, Fahnestock M. Sensory protection of rat muscle spindles following peripheral nerve injury and reinnervation. Plast Reconstr Surg 2009; 124:1860-8.

24. Wang H, Gu Y, Xu J, Shen L, Li J. Comparative study of different surgical procedures using sensory nerves or neurons for delaying atrophy of denervated skeletal muscle. J Hand Surg Am 200I;26:326-3I.

25. Hynes NM, Bain JR, Thoma A, Veltri K, Maguire JA. Preservation of denervated muscle by sensory protection in rats. J Reconstr Microsurg 1997; 13:337-43.

26. Papakonstantinou KC, Kamin E, Terzis JK. Muscle preservation by prolonged sensory protection. J Reconstr Microsurg 2002; 18:173-82.

27. Michalski B, Bain JR, Fahnestock M. Long-term changes in neurotrophic factor expression in distal nerve stump following denervation and reinnervation with motor or sensory nerve. J Neurochem 2008; 105: I 244-52.

28. Sunderland S, Bradley KC. Denervation atrophy of the distal stump of a severed nerve. J Comp Neurol 1950;93:40I-9.

29. Sulaiman OA, Midha R, Munro CA, Matsuyama T, Al-Majed A, Gordon T. Chronic Schwann cell denervation and the presence of a sensory nerve reduce motor axonal regeneration. Exp Neurol 2002; 176:342-54.

30. Martini R, Schachner M, Brushart TM. The L2/HNK-I carbohydrate is preferentially expressed by previously motor axon-associated Schwann cells in reinnervated peripheral nerves. J Neurosci 1994; I4 (II Pt 2):7।80-9I.

31. Yoshimura K, Asato H, Jejurikar SS, Cederna PS, Urbanchek MG, Kuzon WM Jr. The effect of two episodes of denervation and reinnervation on skeletal muscle contractile function. Plast Reconstr Surg 2002; 109:212-9.

32. Zuijdendorp HM, Tra WM, van Neck JW, Mollis L, Coert JH. Delay of denervation atrophy by sensory protection in an end-to-side neurorrhaphy model: a pilot study. J Plast Reconstr Aesthet Surg 2010;63:1949-52.

33. Li QT, Zhang PX, Yin XF, Han N, Kou YH, Deng JX, Jiang BG. Functional recovery of denervated skeletal muscle with sensory or mixed nerve protection: a pilot study. PLoS One 2013;8:e79746.

34. Shea JE, Garlick JW, Salama ME, Mendenhall SD, Moran LA, Agarwal JP. Side-to-side nerve bridges reduce muscle atrophy after peripheral nerve injury in a rodent model. J Surg Res 2014;187:350-8.

35. Magdi Sherif M, Amr AH. Intrinsic hand muscle reinnervation by median-ulnar end-to-side bridge nerve graft: case report. J Hand Surg Am 2010;35:446-50.

36. Hayashi A, Pannucci C, Moradzadeh A, Kawamura D, Magill C, Hunter DA, Tong AY, Parsadanian A, Mackinnon SE, Myckatyn TM. Axotomy or compression is required for axonal sprouting following end-to-side neurorrhaphy. Exp Neurol 2008;2 1 I:539-50.

37. Brenner MJ, Dvali L, Hunter DA, Myckatyn TM, Mackinnon SE. Motor 
neuron regeneration through end-to-side repairs is a function of donor nerve axotomy. Plast Reconstr Surg 2007; 120:215-23.

38. Mackinnon SE, McLean JA, Hunter GA. Direct muscle neurotization recovers gastrocnemius muscle function. J Reconstr Microsurg 1993;9:77-80.

39. Brunelli GA, Brunelli GR. Direct muscle neurotization. J Reconstr Microsurg 1993;9:81-90.

40. Swanson AN, Wolfe SW, Khazzam M, Feinberg J, Ehteshami J, Doty S. Comparison of neurotization versus nerve repair in an animal model of chronically denervated muscle. J Hand Surg Am 2008;33: $1093-9$.

41. Cornbrooks CJ, Carey DJ, McDonald JA,Timpl R, Bunge RP. In vivo and in vitro observations on laminin production by Schwann cells. Proc NatlAcad Sci U SA 1983;80:3850-4.

42. McGarvey ML, Baron-Van Evercooren A, Kleinman HK, Dubois-Dalcq M. Synthesis and effects of basement membrane components in cultured rat Schwann cells. Dev Biol 1984; 105: 18-28.

43. Muir D. The potentiation of peripheral nerve sheaths in regeneration and repair. Exp Neurol 2010;223:102-II.

44. Cederna PS, Youssef MK, Asato H, Urbanchek MG, Kuzon WM Jr. Skeletal muscle reinnervation by reduced axonal numbers results in whole muscle force deficits. Plast Reconstr Surg 2000; 105:2003-9.

45. Ochi M, Kwong WH, Kimori K, Takemoto S, Chow SP, Ikuta Y. Delay of the denervation process in skeletal muscle by sensory ganglion graft and its clinical application. Plast Reconstr Surg 1996;97:577-86.

46. Ochi M, Kwong WH, Kimori K, Chow SP, Ikuta Y. Reinnervation of denervated skeletal muscles by grafted dorsal root ganglion. Exp Neurol I 992; I I8:29I-301.

47. Cederna PS. Discussion: patterns of target tissue reinnervation and trophic factor expression after nerve grafting. Plast Reconstr Surg 2013;131:100 I-3.

How to cite this article: Nghiem BT, Sando $\mathrm{IC}, \mathrm{Hu} \mathrm{Y}$, Urbanchek MG, Cederna PS. Sensory protection to enhance functional recovery following proximal nerve injuries: current trends. Plast Aesthet Res 2015;2:202-7.

Source of Support: Nil, Conflict of Interest: None declared.

Received: 01-02-2015; Accepted: 11-03-2015 